\title{
Chapter 15 \\ The COVID-19 Outbreak and Public \\ Health Issues: An Interdisciplinary Approach
}

\author{
Anavaj Sakuntabhai and Bernard Thomann
}

\begin{abstract}
This chapter aims to present the content of the discussions that took place following he presentations of the workshop "The COVID-19 Outbreak and Public Health Issues: an Interdisciplinary Approach". Discussion focused in particular on how interdisciplinary research can be useful for policy, on how the pandemic changed the way to do research. It was in particular question of research purpose testing, epidemiological surveys, and its related matters such as reliability, technology, methods, samples, ethics. Were also discussed the question of respective weight of social and biological factors in the diffusion of the epidemy and how collective representations could affect information and population trust in public health policies.
\end{abstract}

Keywords Covid19 $\cdot$ Interdisciplinary research $\cdot$ Epidemiology $\cdot$ Life sciences Social sciences $\cdot$ Policy

\section{Introduction}

On December 16 and 17, 2020 the workshop "The COVID-19 Outbreak and Public Health Issues: an Interdisciplinary Approach" took place hosted by Anavaj Sakuntabhai and Bernard Thomann of Institut français de recherche sur le Japon à la Maison franco-japonaise. There were eight presentations during the two days (presenters: Yano, Paul, Sala, Buhnik, Hirota, Ladmiral, Giles-Vernick, and Matsuda), presided over by Thomann and Anavaj. Seven chapters of this book are based on those presentations. ${ }^{1}$ The presentations were followed by a long sequence of discussions, in which many of the coauthors of the presentations participated. This chapter aims to present

\footnotetext{
${ }^{1}$ The program can be found at https://www.mfj.gr.jp/agenda/2020/12/16/rieti/ and https://www. mfj.gr.jp/agenda/2020/12/16/rieti/.
}

\author{
A. Sakuntabhai \\ Institut Pasteur, Paris, France \\ B. Thomann $(\bowtie)$ \\ IFRJ-MFJ, Tokyo, Japan \\ e-mail: thomann@mfj.gr.jp
}

M. Yano et al. (eds.), Socio-Life Science and the COVID-19 Outbreak, Economics, Law, and Institutions in Asia Pacific, https://doi.org/10.1007/978-981-16-5727-6_15 
the content of these discussions, which set the tone of our joint research. The editors of the book express many thanks to the Institut français de recherche sur le Japon à la Maison franco-japonaise for the wonderful workshop.

\section{Covid and the New Need for Interdisciplinary Research. How Interdisciplinary Research Can Be Useful for Policy?}

One of the purposes of our workshop was to build links between different researchers from biological, medical, and social sciences. It is not an easy task because of very different scientific traditions and practices. Bernard Thomann pointed out that we use very different kind of data sources. For example, some researchers used aggregated data, some used individual data. Most data used are qualitative from interviews; when quantifiable, different methods are used. Therefore, it is not easy for researchers to exchange their data (especially data containing personal information), and methods, although we learn a lot when we present our results. Bernard Thomann had questions on how the COVID-19 pandemic had changed the life of researchers. Of course, COVID-19 changed their everyday life. But it also changed the way scientists see their research fields. One year ago, nobody was researching COVID-19 but in this panel, everybody is now working on it. He asked two questions to Shigeru Hirota. His first question was how the COVID-19 pandemic changed the way we practise our field or our discipline? Also, how we realized that we needed more than before an interdisciplinary approach? The second question is about expected results from our researches. Certainly, there are results that we expect and results that we do not expect. Some results could be counter-intuitive. Shigeru Hirota replied that indeed the COVID-19 outbreak had changed our practices and styles of our research. Makoto Yano prepared an interdisciplinary research program more than 4-5 years ago. Now we have to work harder to struggle with this situation, but he was not sure what kind of results we will obtain. He does not know if it will be counterintuitive or not but we have to work harder. Fumihiko Matsuda added that, as compared to France, there is are a much smaller number of patients or people who get infected. Of course, ideally, if they have sufficient number of people who get infected, who had past infection history in the planned study, then they can compare different factors like the social capitals, biological characteristics, and so on. In Nagahama city where they performed longitudinal cohorts, they do not expect to have a large number of people who get infected. But still, they can try to associate social capitals and the change of activities during this COVID-19 pandemic period. They can ask questions about whether individuals have spent more time staying at home or whether their activities have not changed. They can correlate the chance of getting infected with gambling, income, or trust in others. We may have some correlations, which can be used for future policy planning. In this sense, it is crucial to have the social life science study in Nagahama cohort by using the COVID antibody test. This is a very good example 
of a multidisciplinary study studying human beings to take into account the mental activity of people.

Makoto Yano gave his opinion that this particular occasion symbolizes what we will see in the future about our research. Because of COVID-19, we have now really a common interest no matter which field we are in. There are targets that all scientists want to understand: why COVID-19 is spreading badly and strangely in a defined culture, in defined places, and in a defined way. All of us would like to understand the risks from very different viewpoints. He said that this occasion was really wonderful, and that he was very grateful to everybody who gave a presentation in such a way that people who are outside of the field can understand what others are thinking about their interests and their motivations for their own research. Because this kind of occasion was made possible through online conference, we are going to have a totally different type of atmosphere in the international research field, collaborating between different fields.

\section{How Covid-19 Changed the Way to Do Research?}

Olivier Telle from CNRS India raised a question about how COVID-19 had really changed the way we work. As a health geographer, it was very difficult to get access to public health data. During his Ph.D., he spent 1-2 years to convince municipalities and government of India to give him dengue data. Recently, we can get access to all the COVID-19 data online. They have already been geo-referenced, which means that they are already specialized. We therefore have work that has already been done. It is then possible to compare, for example, the diffusion of COVID-19 influence in Japan with other countries.

In the past, it was very difficult for social scientists to access data without collaboration with biologists. Thanks to the COVID-19 pandemic, these datasets are more accessible nowadays. We can test our hypotheses, for example, about social disparities and the links with COVID-19. This is very important for social scientists. It will be a big change to bring social and biological sciences together-although it is not easy and it needs time to explain biology to social scientists and vice versa.

Sophie Buhnik wondered whether researchers at the Institut Pasteur had already studied the possible long-term effects of COVID-19 lockdown measures on cognitive and physical health, especially of the elderly. Recently, there was an article concerning elderly people in Japan who were active. Many policies in ageing societies all promoted elderly to maintain physical health for greater life expectancy. Lockdown measures have significantly diminished the time spent in walking or moving and, therefore, have potential negative effects. Needless to say that there is a lack of communication, which has a detrimental effect especially for people who are in retirement homes, who cannot see their relatives. It has enormous effect on not only their morale but also their cognitive health. They cannot speak and touch their relatives who could visit them in their retirement homes. Are some groups inside the Pasteur Institute already interested in this issue? 


\section{The Question of Research Purpose Testing, Epidemiological Surveys, and Its Related Matters: Reliability, Technology, Methods, Samples, Ethics}

A group made up of Fumihiko Matsuda, Shigeru Hirota, and Makoto Yano studied social factors influencing the spreading of COVID-19 in Nagahama city, which is a medium-sized city. The medical school of Kyoto University, led by Professor Matsuda established a longitudinal cohort for genomic study in the city in 2007. They did the very first baseline study between 2007 and 2010. They have collected chronological and biological data. They have designed a cohort so that many different studies can be added. Fumihiko Hirota and Makoto Yano started this study in 2014. They conducted a questionnaire on social and economic behaviours. They added data on the economic side of the population on top of basic information obtained from a long-time survey. The reason for choosing Nagahama is that it is not very far from Kyoto and people are very supportive to this large-scale cohort.

Sophie Buhnik had a question related to the questionnaire distribution and the respondents. Do you have administrative and local support? How is the questionnaire welcomed by local government? Do you conduct them in cooperation with other organizations? Fumihiko Matsuda replied that researchers from the universities are not allowed to have personal information of the participants. Whenever they conduct a paper-based questionnaire survey, they ask the city of Nagahama and also a nonprofit organization (NPO) that supports the study to make the necessary arrangements to send and to receive the questionnaires so that university staff do not touch/see the envelope with the name and the address of the participants. In short, it is the city and NPO that take care of this administrative procedure, and the research team take care of the research design and analysis.

There is another question related to the cohort. Researchers are not able to survey all the households of the area. So, was there any sampling method to select a quite diverse panel in terms of social background and other characteristics? The study is based on voluntary answers, so what kind of bias can it bring to this cohort of respondents? Shigeru Hirota explained that although the characteristics of the participants have several kinds of bias on gender composition or education, they utilized the benefit of the voluntary basis. If possible, they have to adjust the bias but, first of all, they gather information from the population. The reason why we limit the age group to those under 65 is because the probability of the infection is much higher than the younger and the older age group. Makoto Yano admitted that they expected a very strong bias for a social scientific viewpoint. But at the same time, the beauty of this research is that it is tacked on to the medical and genomic data. It is very unique to associate social behaviours to a cohort with biological and medical data. Fumihiko Matsuda had the cohort data capturing the medical aspects of people, their respondents, over a more than ten-year span. The association study based on this longitudinal medical cohort data is a very interesting thing to do as a social scientist. As a result, from the viewpoint of social science, there is bias. Even if there is bias, 
they get lots of knowledge out of the study in terms of the relationship between life science and social science.

The metropolitan government of Tokyo launched a campaign to assess seroprevalence. They are now recruiting 3000 people to know what the technology will be used for and what type of antibodies will be tested. In the past survey of a serological study, they used the antibody test kit manufactured by Abbott or Roche. Both of them target two antigens of virus. They found seropositive individuals, two people who are seropositive in both tests and the other five or six are seropositive for one test and not for the others. The accuracy of the result is not that satisfactory. The antibody test technology developed by Institut Pasteur is much more sensitive and specific.

What is the sample size? It would be interesting to have serology data of large population where there was a low transmission level like in Japan. This study will help us to assess better the level of asymptomatic infection and to understand better who transmits the virus. Fumihiko Matsuda explained that they will start the study in January with a target sample size of 3000 in Nagahama city. In this community-based cohort, they will test once. In Kyoto University Hospital, they will follow up with around 1000 healthcare workers. They will follow them up three or four times- the first survey in January, the second in March, the third in June, and the fourth next autumn. They continuously check the health status of healthcare workers. They do not have to set up a new sample collection, but they can use the samples that are collected for some other purpose.

They will do the antibody test and social science study in parallel. They will start with the sociological survey. The measurement of antibody will take place later. Once they get the samples, they can measure the samples at any time, as we can keep the materials in good condition.

Lastly, there is a question on the ethics of using people movement data from the Facebook application. Samuel Benkimoun explained that Facebook is aware of this concern. They provided the data in an aggregated form. There is no individual data and no data of underage individuals (less than 18 years old). And even more important, the data is only collected from people who agreed to share their location with the Facebook application. For example, they provided data in the form of a grid. They did not provide data if there are fewer than ten people inside a grid. The reason is that this data is too precise and cannot guarantee the privacy of the users.

\section{The Question of Respective Weight of Social and Biological Factors in the Diffusion of the Epidemy}

As was emphasized above, the contribution of the human and social sciences to the understanding of the epidemic was one of the main themes of the discussion. Makoto Yano's work on the US shows that people's attitudes towards risk can vary considerably from one country to another, and that, whatever the location, getting people to accept social distancing or wearing masks is a particularly difficult challenge for 
the authorities. All the more so as these very behaviours can be a political positioning issue and blur the message of scientific experts. Indeed, President Trump has encouraged behaviours of defiance of protective measures advocated by scientists, by highlighting the value of courage. It has been pointed out that Florida, led by a Republican close to Donald Trump, has particularly suffered from Covid 19. According toMakoto Yano, if the message of the president and the governor is convergent, it has a positive effect on people's behaviour.

The learning effect of prophylactic methods by the populations confronted with the pandemic is thus blurred by a large number of factors that make the contribution of social sciences particularly valuable. Thus, one example raised by the discussion beyond the influence of political messages is the influence of city size. As Samuel Benkimun highlights, an important point is how these different factors interact with each other-for example, the learning capacity of populations with the size of the city. In response to this question, Makoto Yano says that this relationship is complex to interpret. At first sight, the larger the city, the stronger the epidemic. Thus, COVID19 was seen as primarily a disease of large cities, especially those in New York and New Jersey. But, on the other hand, what the data shows is that if you look at a particular region, the larger the city, the lower the number of cases per capita. This may seem surprising, but it confirms his initial conjecture that people have come to learn, struck by what was happening in New York, that the more urban the environment, the more careful you have to be. However, this becomes even more complicated when we realize that in the really densely populated areas, this effect disappears. It then appears that the factor of a large, densely populated city becomes more powerful than the factor of the population learning to take protective measures.

Bernard Thomann asked if the learning factor itself should be relativized since over time it does not have as much influence - many people get tired of the mask and social distancing, as is the case today in France. However, Makoto Yano believes that it should be interpreted for a given moment, the beginning of the epidemic, since the data used was collected on April 15, 2020. At that time, there was still a much purer effect between learning and size of the city than today where there are more and more factors to take into account. He feels that another study should be conducted today.

Another social factor to consider is the healthcare system. In response to a question from Richard Paul, Remi Scoccimaro mentioned the fact that hospitalization can cost a lot of money in Japan and that this can influence people's behaviour. In relation to this question of the economic capital factor, Bernard Thomann asked Remi Scoccimaro if his thesis that the richest neighbourhoods are also those most affected by the epidemic should not also take into account the age factor. Indeed, some of the richest neighbourhoods, especially the neighbourhoods with large luxury mansions, are also inhabited by relatively young people who protect themselves less, while in less affluent neighbourhoods, on the outskirts, there is an older population. Rémi Scoccimaro's answer is that this phenomenon may be true, but not everywhere. In the residential part of western Tokyo, there are clusters of neighbourhoods that are both rich and old. 
Guillaume Ladmiral asked a methodological question about the way in which the wealth of a district is determined and thus on what condition it is really possible to make a link between the level of contamination and the level of wealth. For Rémi Scoccimaro, it is the price of land. Here again, as for other indicators, one must be careful because, in Tokyo, people without economic means can live in the middle of rich districts, such as Shibuya. However, with the destruction of the oldest houses, which were often inhabited by elderly people, this phenomenon is more and more marginal. Thus, according to him, the price of land is a good indicator, which can be more easily cross-checked with the indicators of the level of contamination.

Finally, another characteristic of these rich districts, which may explain why they are more contaminated, is that they are inhabited by rich foreigners who travel a lot. The Japanese living in these areas are themselves more mobile than the rest of the population. Indeed, as Guillaume Ladmiral points out, the National Institute of Infectious Diseases has shown that the strain coming from Wuhan in China disappeared quite early, at the end of March. So the strains that caused the next waves of infection came from the US and Europe. And they were mainly introduced by Japanese people coming back from vacation in March and April or professionals coming back from their missions abroad. For example, if you look at Aomori Prefecture which had very few cases, the virus was introduced there by a group of retirees returning from Spain and Kanto, and by students returning from the US. The complexity resulting from the interaction between the different factors is underlined by Olivier Telle, for whom a simple factor - the elderly are less affected for example — can hide a more complex phenomenon, notably that the elderly live more in rural areas. However, Guillaume Ladmiral, who in his study tries to take into account a very large number of factors in his analysis of social determinants, points out that this complexity is not always possible to deal with satisfactorily since data are not always available.

While there are many social factors in the spread of the epidemic that can be captured by various social science research methods, they coexist with biological factors. And it is difficult to say, as is the case in big western cities where social (for example economic precarity) or biological (ethnic belonging) factors prevail. Indeed, according to Anavaj Sakuntabhai, what also interests scientists is that the number of severe cases and the number of deaths are different depending on the ethnic group. Like other geneticists, he is very interested in knowing whether the genetic background of humans can explain this. It can be hypothesized that certain ethnicities are predisposed to severe cases and deaths from COVID-19. A recent study of human genetics showed that a region of chromosome 3 , inherited from both father and mother, appeared to predispose to severe COVID-19 infection and death. And, currently, this segment of the genome is more prevalent in south Asia (India, Bangladesh), where you can see a lot of severe cases and deaths, but not in east Asia (Japan and China), where there is a very small percentage of this segment of the genome. 


\section{Epidemic, Collective Representations and Social Consequences}

While researchers in the humanities and social sciences can rely on a whole range of statistical data to try to identify the social factors that, alongside the more purely biological factors, make it possible to identify the dynamic of the epidemic, they must also consider the collective representations that populations have of the disease. These representations obviously influence the way in which public health policies are received by these populations.

In times of epidemics, it is of course necessary to question the way in which the risk is perceived by the population. According to Samuel Benkimou, who is currently living in India, the political message of mobilization against the epidemic has to face the fact that a significant part of the population is not convinced that COVID-19 is a real threat. There is also a significant amount of doubt mixed with local beliefs and alternative medicines that coexist with official medical science, and religiously tinged messages that contribute to a somewhat blurred view of what constitutes the threat and what needs to be done. On the other hand, in rural areas, very few people are seen wearing masks because they are convinced that their difficult past will give them some form of resistance or perhaps cross-immunity with other diseases to which they have been exposed. While it has been said that in Japan it was the higher income people who seemed to be less afraid of the virus because they had easier access to medical care, in India it would be quite the opposite. People at the top of the social ladder protect themselves and can isolate themselves in closed residential communities, forbidden to all foreigners. In contrast, the majority of people from low-income backgrounds are more exposed. Since they often work in the informal sector- $80 \%$ of people in India have no employment contract - they depend on their work for their livelihoods regardless of the threat and risks they may face. One audience member suggested another important factor that explains why wealthy people in Japan seem less cautious about the pandemic. The wealthiest people can afford to be tested - they have better access to testing than an ordinary worker. And this may be the reason why, in the first wave, there was a concentration of positive tests in the richest parts of Tokyo, as Rémi Scoccimarro has shown. But this may also be changing over time. The unions stress the importance of testing essential workers as well and asking employers to test their employees regularly, every two weeks.

For Rémi Scoccimaro, the phenomenon of stigma is important for understanding how different social groups react to the pandemic. This issue of stigma is critical in Japan for less affluent people, who need the support of their community. Indeed, once it is known that someone is infected within the community, they may face a very high level of stigma. In Japan, even nurses and doctors face this phenomenon. And if someone lives in a typical Japanese neighbourhood, where solidarity among people is very important, and if they lose the benefit of that solidarity, they lose a lot. So, in a way, that individual has no interest in getting tested or revealing that he has been infected and, especially, they take a lot of precautions not to get infected. This is something that wealthier people do not worry about as much because they 
can do without the community and they do not live in the same model of city. This issue of stigma is known in France, but in Japan it is something really structural. It also emerged for people who were irradiated after the Fukushima accident. The fear of stigma can be stronger than the fear of the virus itself. For example, some sick people who call an ambulance ask the ambulance to turn off its alarm so as not to alert the neighbourhood.

Adrienne Sala adds that the problem of high discrimination in Japan is also linked to the fact that there is less contamination, or at least known contamination, thanks to the PCR test. But in France for example, where there is a higher level of contamination and PCR testing, most people know infected people around them. This normality implies lower discrimination. For Anavaj Sakuntabhai, in Japan, the perception is still far from that of an ordinary disease. For Richard Paul, however, if everyone in France knows someone who has had COVID-19, there is still fear.

Another question raised is that of the Japanese relationship to hygiene. The high level of hygiene in Japan is often mentioned, for cultural or historical reasons, linked to the long-standing importance of hygiene policies. Nevertheless, Rémi Scoccimaro believes that we must be careful with cultural stereotypes that can be misleading. Japanese people are not uniformly more hygienic. He even points out that certain Japanese habits, such as the one for several people to take a bath successively without changing the water, are not particularly hygienic.

The human and social sciences not only highlight the social phenomena and representations that influence people's behaviour, such as proximity to a political party, membership of a social class, stigmatization, education, perception of risk, relationship to hygiene, but also certain consequences of the epidemic in society. Bernard Thomann reports a very worrying statistic in Japan at the moment: a suicide rate that is increasing for women much more than for men. Makoto Yano said that South Korea has a similar problem, but that he has not seen similar phenomena in other countries. In his opinion, this phenomenon is probably partly linked to the poor working conditions of many women, non-regular workers who are the priority victims of lay-offs. Rémi Scoccimarro adds that this is a surprising phenomenon given that the suicide rate in Japan has been decreasing for ten years. However, he created maps on suicide in Japan, and also after the Fukushima accident, and this allowed him to make some comparisons. In Asia, women are more suicidal than in other countries and this is a particular feature in Japan, Korea, Taiwan, and China. Especially in rural areas of China, the suicide rate of women is higher than in east Asian countries. The cause is not only professional; with the lockdown and telecommuting, the husband is more at home, which implies a greater burden on the wife. It may be interesting to find out in which part of Japan women commit suicide more. Usually, it is in the north, in the Akita prefecture, that the mental pressure on the group is the highest.

It seems that the curve is higher for women between 25 and 35 years old in Asia than it should be if we compare with other regions of the world. But it would be interesting to have other statistical elements, such as the profession of the person who committed suicide. Adrienne Sala adds that there is a child abuse prevention program in Japan that usually presents data on suicide of single mothers, which is very high compared to the general suicide rate. So during the crisis, like the health 
crisis that led to an economic crisis, it exacerbated the already existing trend in Japanese society. But these economic causes were exacerbated by the crisis and the lack of support from the government or other actors.

\section{Information and Trust}

Makoto Yano recalled that what was well demonstrated during the presentations and discussions was that from a social science perspective, taking into account culture and economic factors is important to study the behaviour of populations in the face of the epidemic. But what is also particularly important is how information is delivered in society. From this point of view, Makoto Yano thinks that politicians need to give a much clearer message. They have been very confused, at least in Japan he thinks, and he wonders if it has been the same in other countries-especially if you think about the "Go To Travel" campaign, which subsidized travel while at the same time many people were being advised not to travel.

The lack of trust in official statistics seems to be particularly high in Japan, as Rémi Scoccimarro points out. The general idea among the population is that if there are few cases in the archipelago, it is because there are so few tests. For Makoto Yano, in addition to the low number of tests, there are many people who do not want to go to hospital, especially if their condition is not really serious, especially since declaring oneself ill is a rather cumbersome process. As far as deaths are concerned, because the information is public, even if there is an underestimation, the possibilities of manipulating the information are relatively limited, according to him. Richard Paul points out that in other countries too the number of deaths officially recorded probably does not really reflect the real figures. What specialists rely on is the excess mortality figures for all causes among people over 60. If there is excess mortality, even if people have not been tested, these deaths are attributable to COVID-19.

This question of trust is also found in the adherence of the population to tracking applications. Bernard Thomann points out that, depending on the country, the download rate of tracking applications can be very different. For example, in Singapore or Iceland, some data show that about $40 \%$ of the population download these tracking applications. In France, the rate is only 3\%, and Japan is in between these two extreme cases. According to Anavaj Sakuntabhai, there may be a reluctance to download the application since it is not clear what the consequences will be for oneself and one's entourage if one is identified as a contact case. The official Japanese tracing application actually coexists with a multitude of applications developed, both locally and nationally, by citizens. It even happens, as Sophie Buhnik points out, that the authors of COVID-19 information sites work for local governments. These operators work voluntarily for the prefecture, and help the local government to disseminate information to citizens in a crisis context. But Adrienne Sala notes that, although civil society is very present today in informing the population, this movement is unfortunately counterbalanced by the lack of transparency in the decision-making process within the committee of experts linked to the Ministry of Health, Labour and Social Affairs. 
According to Richard Paul, the implementation of tracking applications in Europe is also problematic and very confusing. The EU wanted a kind of universal application so that people coming from Italy, for example, could detect if they were a contact case for someone in France. However, according to Richard Paul, if we add to the European and national levels also the regional level, all these spatial discontinuities in terms of information, but also of political messages, of interventions make the situation chaotic. Moreover, as Sophie Buhnik also points out, the profusion of information raises another problem, that of the notion of infodemics - that is to say, an overload of information that makes it difficult to sort through all the available figures and causes fatigue in the population.

Another barrier is the digital divide, as one viewer noted in his question. However, Sophie Buhnik believes that even though programmers are mostly men, young Japanese women have as good a university education as their male counterparts, sometimes even better, and are equally capable of reading data. This digital divide could be about age difference; however, electronic literacy among people over 60 and 70 is about $50 \%$, much better than in other countries. This divide can be to some extent geographical, with more urbanized departments having stronger collectives of programmers.

If a segment of the population is in an attitude of mistrust, it is because, as one contributor expressed it, we are in a period where multiple tools are being put in place to progressively follow the population and collect more and more information on individuals. For now, this is being done for health motivations, which still makes it acceptable to many. But is this not one more step in the direction of a control and surveillance society? Once we are out of the context of the epidemic and can think that these tools are no longer useful, will surveillance really stop? Especially, as Guillaume Ladmiral expresses it, we can legitimately ask ourselves if this intrusive technology is really necessary to fight the pandemic effectively. For example, there has been no mass surveillance or large-scale application in Japan and Taiwan, although the figures there are quite good.

The issue of trust in the context of the profusion of information applications on COVID-19 actually applies to all sorts of areas. Bernard Thomann believes that what is at stake is of course political, civil, and social citizenship, but also what social scientists and researchers are increasingly calling biological citizenship. There is a fear of citizens in the face of all the new technologies that can change their lives, or even their biological nature. What is very interesting, for example, is that people are afraid that these new messenger RNA vaccines will change our genetic makeup.

How people can trust or not trust these biopolitical policies really interacts with how not only the government, but also the scientific community, protects this biological citizenship. Anavaj Sakuntabhai asks Fumihiko Matsuda about the Nagahama city study presented during the workshop, how the authors of this study comply with data protection, ethics, and Japanese law. According to Fumihiko Matsuda, in Japan such a project must be submitted to the ethics committee for review, and this takes a long time. But it is clearly written in a government directive that if the study is urgent, it must go through the ethics committee, but in the case of Kyoto University Medical School, the dean or the president of the hospital can approve the study before getting 
approval from the ethics committee. So, if the dean or the president of the hospital thinks it is urgent and necessary and can be started quickly, the investigation can start. However, informed consent must be obtained from the participants. In the case of the Nagahama study, a questionnaire is sent out and, if the residents return it, it means that they are willing to participate in the study.

Anavaj Sakuntabhai MD, D.Phil. is a medical doctor from Thailand. After his Ph.D. on human molecular genetics at the Wellcome Trust Centre for Human Genetics, University of Oxford in 2000, he joined the Institut Pasteur to develop a programme on genetics of infectious diseases. He created the research unit of Functional Genetics of Infectious Diseases at Institut Pasteur in 2010 focusing on genetic susceptibility to dengue infection. He received Prix Dusquense in 2016 and i-Lab Grand Prix award from the French national challenge of innovation organized by the French Ministry of research in 2020 for development of new pentavalent dengue and Zika T cell vaccine. He coordinated European FP7 project on Dengue Framework for Resisting Epidemics in Europe (DENFREE). He was involved in investigating two recent global outbreaks of infectious diseases, Ebola and Zika. Currently, he is a coordinator of the Pasteur International Center for Research on Emerging Infectious Diseases (PICREID)—-supported by the NIH. The project is implemented in west and central Africa and southeast Asia, linking large observational multicentre cohort studies with basic scientific research and leading to increased preparedness for new epidemic threats in the region. Recently, he was appointed as the director of Institut Pasteur Japan office to establish a transdisciplinary network on emerging infectious diseases between Japan, France, and countries in the Indo-Pacific region.

Bernard Thomann is an historian, Director of Institut français de recherches sur le Japon à la Maison franco-japonaise (MEAE/CNRS), and professor at the Institut National des Langues et Civilisation Orientales in Paris. He is a former visiting researcher at the University of Tokyo and visiting professor at the Tokyo University for Foreign Studies. His research interests include the history of health and social policies in modern and contemporary Japan, labour history, the history of occupational diseases, and the health and social history of the mining industry. His latest book is La naissance de l'Etat social japonais: Biopolitique, travail et citoyenneté dans le Japon impérial (1868-1945) (2015). He is scientific coordinator for the research programme funded by the French Agence Nationale de la Recherche (ANR): Growth and Forms of Employment: a Eurasian Comparison of Employment Insecurity (EURASEMPLOI) (2016-2022) and participated in the project "Transnational study of an exemplary occupational disease: silicosis and occupational health in France and the industrialised countries" (ANR "SANTE-ENVIRONNEMENTTRAVAIL PROGRAMME (SEST)” 2007-2010). 
Open Access This chapter is licensed under the terms of the Creative Commons AttributionNonCommercial-NoDerivatives 4.0 International License (http://creativecommons.org/licenses/bync-nd/4.0/), which permits any noncommercial use, sharing, distribution and reproduction in any medium or format, as long as you give appropriate credit to the original author(s) and the source, provide a link to the Creative Commons license and indicate if you modified the licensed material. You do not have permission under this license to share adapted material derived from this chapter or parts of it.

The images or other third party material in this chapter are included in the chapter's Creative Commons license, unless indicated otherwise in a credit line to the material. If material is not included in the chapter's Creative Commons license and your intended use is not permitted by statutory regulation or exceeds the permitted use, you will need to obtain permission directly from the copyright holder.

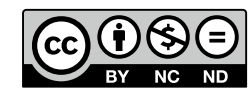

